\title{
Efecto de la adición de agua electroactivada a la bebida de bovinos de engorde a corral (feedlot), en un establecimiento de la provincia de Buenos Aires
}

\section{Effect of electrochemically activated water addition to the drinking water of feedlot cattle of Buenos Aires province}

\author{
Pellicer KE ${ }^{1 *}$, Brusa V¹, de la Torre JH¹, Real D², Silvestrini MP²,3, Bainotti \\ CA $^{2}$, OSElla OE ${ }^{4}$, COPES $\mathbf{J}^{1}$
}

1. Laboratorio de Microbiología de Alimentos, Cátedra de Tecnología y Sanidad de los Alimentos; 2. Cátedra de Epidemiología Aplicada. Facultad de Ciencias Veterinarias, Universidad Nacional de La Plata. 3. Instituto de Investigación en Ciencias de la Salud, Escuela Superior en Ciencias de la Salud. Universidad Nacional del Centro de la Provincia de Buenos Aires. 4. Actividad privada.

$$
\text { * Correo electrónico de la autora de contacto: pellicerk@fcv.unlp.edu.ar }
$$

\begin{abstract}
Resumen
El objetivo general del trabajo fue evaluar el efecto del consumo de agua electroactivada (AE) en bovinos de engorde a corral (feedlot); los objetivos particulares fueron: 1. determinar la presencia de E. coli O157 y de Salmonella spp. en materia fecal y 2. determinar su eficiencia como promotora de crecimiento. Se utilizaron 150 bovinos divididos en 4 lotes (L). El L1 fue tratado con 0,3 \%, 1,5 \% y 3,0 \% de AE; el L2 con 0,3 \% y 1,5 \%; el L3 con o,3 \% y el L4 no fue tratado. En cada visita se pesaron los animales y se obtuvieron muestras de materia fecal, y en la última también de sangre. En materia fecal se determinó la presencia de E. coli O157 y de Salmonella spp. En sangre se determinaron: $\mathrm{pH}$, bicarbonato, sodio, potasio, cloro y hemoglobina total. Para el procesamiento de datos estadísticos se utilizó el programa Infostat. Con respecto a la ganancia de peso, la tendencia a favor del L2 y L3 a los 6o días de tratamiento, podría deberse al efecto promotor de crecimiento por el consumo de AE. Las muestras de materia fecal resultaron positivas para E. coli O157 en 3,3 \% para el L2 a los 60 días, y para Salmonella spp. en 3,3 \% y 1,6 \% para los L3 y L4 a los 90 días de tratamiento, respectivamente. No se evidenciaron alteraciones en los valores de referencia de los parámetros sanguíneos evaluados. La adición de AE en el agua de bebida de bovinos de feedlot podría ser utilizada como promotora de crecimiento.
\end{abstract}

\section{Palabras clave}

agua electroactivada, feedlot, bovinos, E. coli, Salmonella spp., promotor de crecimiento

\begin{abstract}
The general objective of the work was to evaluate the consumption of electroactivated water (AE) in feedlot cattle; the particular objectives were: 1. to determine the presence of E. coli O157 and Salmonella spp. in feces and 2. determine its efficiency as a promoter of growth. One hundred and fifty animals were grouped into 4 lots (L). The L1 was treated with $0.3 \%, 1.5 \%$ and $3.0 \%$ of EAW; L2 with $0.3 \%$ and $1.5 \%$; L3 with $0.3 \%$ and L4 was not treated. In each of the 4 visits to the feedlot the animals were weighed and fecal samples were taken. Also, in the 4th visit, blood samples were taken. Fecal samples were analyzed to determine the presence of E. coli O157 and Salmonella spp. In the blood samples, $\mathrm{pH}$, bicarbonate, sodium, potassium, chlorine, and total hemoglobin were determined. Data were analyzed using Infostat software. With respect to weight gain, tendency in favor of L2 and L3 after 60 days of treatment could be due to the growth promoter effect of EAW consumption. Fecal samples were positive for E. coli O157 in 3.3\% for L2 at 60 days, and for Salmonella spp. in 3.3\% and 1.6\% for L3 and L4 after 90 days of treatment, respectively. No alterations were observed in the reference values of the evaluated blood parameters. The addition of EAW to the drinking water of feedlot cattle could be used as a growth promoter.
\end{abstract}

\section{Key words}

electroactivated water, feedlot, cattle, E. coli, Salmonella spp., growth promoter

Fecha de recepción: 06/03/2018

Fecha de aprobación: 24/05/2018
ANALECTA VeT 2018; 38(1): 9-15

Impresa ISSN 03655 14-8 Electrónica ISSN 1514-2590

doi.org/10.24215/15142590eo20 


\section{Introducción}

El agua electroactivada (AE) es un producto obtenido por medio del pasaje de una solución saturada de cloruro sódico diluida en agua de red, a través de una celda de electrólisis diafragmática. Las soluciones producidas se llaman fracción anódica y catódica, por el signo de carga del electrodo en el que se sintetizan. De esta forma se consigue una solución desinfectante (fracción anódica) a partir de agua corriente, sin aditivos químicos, por lo que no hay necesidad de manipular productos potencialmente peligrosos tales como el cloro. La fracción anódica (AE propiamente dicha) obtenida en este proceso es ácido hipocloroso, de $\mathrm{pH}$ neutro y con potencial Redox de +850 $\mathrm{a}+1000 \mathrm{mV}$.

Los promotores de crecimiento son frecuentemente utilizados en la producción animal para mejorar la ganancia diaria de peso. En general son productos antibióticos y/o bactericidas y traen aparejadas algunas desventajas como por ejemplo la resistencia antimicrobiana (Hughes \& Heritage). El AE ha sido utilizada como promotora del crecimiento ofreciendo buenos resultados en pollos de engorde y también se utilizó para mejorar la calidad de la leche en vacas de tambo (Holcroft, 2003; Ferguson et al., 2008). A diferencia de los promotores de crecimiento tradicionales, el $\mathrm{AE}$ es un producto antimicrobiano natural, innovador y eficaz obtenido con una tecnología simple y de bajo costo (Tabernero de Paz et al., 2013).

El AE se plantea también como una alternativa a ser utilizada como higienizante del agua de bebida y como agente de limpieza y desinfección de las instalaciones ganaderas (Bartolomé et al., 2011; Envirolife, 2017). Diferentes trabajos han demostrado el efecto antimicrobiano del AE siendo eficaz, segura, fácil de manipular, relativamente barata y ecológica (Botta et al., 2018; FSIS, 2017; Perry \& Yousef, 2011). Estudios previos demostraron que el $\mathrm{AE}$ es altamente efectiva en la eliminación de Escherichia coli $\mathrm{O}_{157}: \mathrm{H}_{7}$, Salmonella enteritidis y Listeria monocytogenes, lo que indica su potencial aplicación para la descontaminación de los alimentos (Venkitanarayanan et al., 1999). Su eficacia no es provocada por el desencadenamiento de reacciones químicas, sino que actúa como simple portador del agente activo (ácido hipocloroso) ya estabilizado.

Los objetivos del control microbiológico de los alimentos son asegurar la inocuidad alimentaria y estimar la vida útil y estabilidad del alimento. Según la Organización Mundial de la Salud (OMS), la inocuidad alimentaria se define como "la garantía de que los alimentos no causarán daño al consumidor cuando se preparen y/o consuman de acuerdo con el uso a que se destinan". E. coli productor de toxina Shiga (STEC) y Salmonella spp. son bacterias patógenas que han sido asociadas con infecciones en humanos debido al consumo de productos alimenticios de origen bovino (Caprioli et al., 2005; Heriksatd et al., 2008). STEC O157 es un agente patógeno emergente asociado a enfermedades transmitidas por alimentos (ETA) (EFSA, 2009). STEC produce una amplia variedad de afecciones humanas: casos esporádicos de diarrea leve autolimitante o brotes de diarrea, colitis hemorrágica, púrpura trombótica trombocitopénica o síndrome urémico hemolítico (SUH) (Karmali et al., 2010). Los rumiantes en general, y el ganado bovino en particular, han sido identificados como el mayor reservorio de STEC (Ferens \& Hovde, 2011). La contaminación de las carcasas puede ocurrir durante el procesamiento en el frigorífico (O’Brien et al., 2005). La mayoría de los animales a partir de los cuales se han aislado cepas de STEC son portadores asintomáticos de la bacteria. Sin embargo, hay excepciones como es el caso de los neonatos bovinos en los cuales cepas $\mathrm{O} 157: \mathrm{H}_{7}$ pueden producir enterocolitis (Dean-Nystrom et al., 1997).

Salmonella spp. es una bacteria patógena responsable de ETA. Diferentes estudios han demostrado la presencia de Salmonella spp. en heces de bovinos (Ramoneda et al., 2013; Agga et al., 2016; Smith et al., 2016). La salmonelosis es un importante problema de salud pública en todo el mundo. Esta enfermedad ha sido asociada con el consumo de alimentos contaminados, principalmente de origen animal (aves, bovinos y cerdos), como también por el contacto directo con animales infectados (Bou-chrif et al., 2009; Fey et al., 2000; Bartholomew et al., 2004; Roy et al., 2001). Los animales enfermos y los portadores asintomáticos pueden eliminar Salmonella spp. a través de las heces y esta bacteria puede sobrevivir durante largo tiempo en el ambiente (Wray \& Wary, 2000).

El objetivo general del trabajo fue evaluar el efecto del consumo de agua electroactivada (AE) en bovinos de feedlot; los objetivos particulares fueron: 1. determinar la presencia de E. coli O157 y de Salmonella spp. en materia fecal y 2. determinar su eficiencia como promotora de crecimiento.

\section{Materiales y métodos}

\section{Animales}

El trabajo experimental se realizó en un feedlot ubicado en el partido de General Belgrano, provincia de Buenos Aires, entre los meses de septiembre y diciembre del año 2015. Se utilizaron 150 bovinos raza Aberdeen Angus, que arribaron al establecimiento en ayunas, 12 horas previas al comienzo del ensayo. Todos los animales provenían de un campo de recría, pertenecientes a la misma empresa y fueron agrupados al azar en 4 lotes. Los lotes $(\mathrm{L})$ fueron homogéneos en cuanto a peso, estado general y conformación, tanto intra como inter lote al inicio del experimento (Día o). El peso vivo promedio de los animales al comienzo del estudio fue de 260 kilos. Para determinar el 
efecto promotor de crecimiento del $\mathrm{AE}$, en cada visita al establecimiento se pesaron todos los animales de un mismo lote en una báscula en grupos de 5 para determinar la ganancia de peso mensual.

Los L1 $(n=30)$, L2 $(n=30)$ y L3 $(n=30)$ se sometieron a diferentes concentraciones de $\mathrm{AE}$ adicionada al agua de bebida (Tabla 1) y el L4 $(\mathrm{n}=60)$ se utilizó como lote control sin tratamiento. El suministro de agua de los corrales que ocuparon los lotes fue a través de un tanque en el cual se dosificó el porcentaje de AE correspondiente para cada mes de tratamiento, con una cañería de alimentación de los bebederos con una llave exclusa en cada caso. Los corrales contaron con otro bebedero secundario al cual se suministró agua sin tratar cuando no correspondiera el tratamiento.

Tabla 1. Esquema de tratamiento con diferentes concentraciones de agua electroactivada en el agua de bebida de bovinos en feedlot

\begin{tabular}{llll}
\hline Lote $(\mathrm{n}=)$ & Día 0 & Día 30 & Día 60 \\
\hline $1(30)$ & $0,3 \%$ & $1,5 \%$ & $3,0 \%$ \\
$2(30)$ & $0,3 \%$ & $1,5 \%$ & $0,0 \%$ \\
$3(30)$ & $0,3 \%$ & $0,0 \%$ & $0,0 \%$ \\
$4(60)$ & - & - & - \\
\hline
\end{tabular}

El AE que se utilizó para el presente estudio fue elaborada por un equipo de origen ruso provisto por la empresa Let Americana, que se encuentra en el Laboratorio de Microbiología de los Alimentos, Facultad de Ciencias Veterinarias, UNLP.

Se realizaron 4 visitas al establecimiento en el periodo de estudio. En cada visita se obtuvieron muestras individuales de materia fecal del recto de todos los bovinos y se colocaron en bolsas plásticas estériles, identificadas para cada animal. Las muestras fueron transportadas refrigeradas al Laboratorio de Microbiología de los Alimentos, Facultad de Ciencias Veterinarias, UNLP, donde fueron procesadas dentro de las 24 horas de recolectadas.

\section{Análisis microbiológicos y PCR}

Para el aislamiento de E. coli O157 se realizó un primer paso de enriquecimiento selectivo. Diez gramos de muestra se colocaron en una bolsa de muestreo estéril y se le adicionaron $90 \mathrm{ml}$ de caldo tripticasa soya modificado con novobiocina (8 $\mathrm{mg} \mathrm{L}^{-1}$ ) (Acumedia Manufactures Inc., Lansing, Michigan, EE. UU.). Las muestras fueron incubadas a $42{ }^{\circ} \mathrm{C}$ durante 24 horas. Luego de la incubación, a partir de $1 \mathrm{ml}$ del caldo de enriquecimiento, se realizó la técnica de separación inmuno- magnética y posterior siembra en estría por agotamiento en placas de agar MacConkey sorbitol suplementado con telurito de potasio y cefixime (SMAC-CT, Acumedia Manufactures Inc., Lansing, Michigan, EE.UU.) y agar SD-39 (Acumedia Manufactures Inc., Lansing, Michigan, EE.UU.). Las placas fueron incubadas durante 24 horas a $37^{\circ} \mathrm{C}$. Luego de la incubación, a partir de cada placa se seleccionó un máximo de 30 colonias típicas y se sembraron en una placa cuadriculada de SMAC-CT. Las placas fueron incubadas durante 24 horas a $37{ }^{\circ} \mathrm{C}$. Luego de la incubación se armaron 3 conjuntos de 10 colonias cada uno, a los que se les realizó la extracción de ADN mediante ebullición a $100{ }^{\circ} \mathrm{C}$ durante 15 minutos, en $150 \mu \mathrm{l}$ de buffer Tritón X-100 al $1 \%$ en buffer TE $1 \mathrm{X}$ [10 $\mathrm{mM}$ TrisHCl (pH 8,o); 1 mM EDTA (pH 8,0)]. Posteriormente, los tubos fueron centrifuga-dos a 10000 rpm durante 5 minutos para sedimentar los restos celulares. Los extractos de ADN de los conjuntos fueron analizados mediante PCR múltiple para la detección de los genes stxi, stxa y $r f b_{\mathrm{O}_{157}}$ (Leotta et al., 2005). Para identificar la colonia $s t x / r f b_{\mathrm{O}_{157}}$, se analizó cada colonia del conjunto positivo mediante PCR múltiple. Se utilizaron tres pares de oligonucleótidos cebadoes, un par para amplificar un fragmento del gen stx1, correspondiente a la subunidad B de la toxina Stx1, otro par para amplificar un fragmento del gen stx2, correspondiente a la subunidad A de Stx2 y el tercer par para amplificar un fragmento del gen rfbO157 correspondiente a una proteína involucrada en la síntesis del lipopolisacárido O157. Como control positivo se utilizó una cepa control E. coli EDL 933.

Para el aislamiento de Salmonella spp. se realizó un primer paso de pre-enriquecimiento. Diez gramos de muestra se colocaron en una bolsa de muestreo estéril y se le adicionaron $90 \mathrm{ml}$ de agua peptonada tamponada estéril (APB) (Acumedia Manufactures Inc., Lansing, Michigan, EE.UU.). Las muestras fueron incubadas a $37^{\circ} \mathrm{C}$ durante 24 horas. Luego de la incubación se realizó el enriquecimiento selectivo colocando $1 \mathrm{ml}$ del caldo de pre-enriquecimiento en tubos con 10 $\mathrm{ml}$ de caldo tetrationato (Acumedia Manufactures Inc., Lansing, Michigan, EE.UU.) y $100 \mu \mathrm{l}$ del caldo de pre-enriquecimiento en tubos con $10 \mathrm{ml}$ de caldo Rappaport (Acumedia Manufactures Inc., Lansing, Michigan, EE.UU.).

\section{Muestreos sanguíneos y análisis bioquí- micos}

En la última visita se recolectaron muestras de sangre mediante punción de la vena coccígea de 5 animales de cada uno de los lotes L1, L2 y L3, y de 10 animales del L4 $(n=25)$. En las muestras de sangre se determinaron 6 parámetros fisiológicos para verificar alteraciones en los valores de referencia por el consumo de agua con adición de AE. Los parámetros evaluados fueron: 
$\mathrm{pH}$, bicarbonato (HCO3), sodio (Na), potasio (K), cloro $(\mathrm{Cl})$ y hemoglobina total (tHB). Para las determinaciones se utilizó el equipo OPTI $\AA$ CCATS2 Blood Gas and Electrolyte Analyzer (IDEXX Laboratories, Inc., Atlanta, Georgia, EE.UU.).

\section{Análisis estadístico}

Para el análisis estadístico, las variables categóricas se resumieron mediante porcentajes, mientras que las variables continuas normalmente distribuidas se presentaron como promedios \pm desviaciones estándar. Se analizaron con la prueba de Análisis de Varianza (diseño no balanceado) y la prueba para el contraste de hipótesis a posteriori en caso de presentar diferencias significativas. Para el procesamiento de datos se utilizó el programa Infostat (http://www.infostat.com.ar/).

\section{Resultados}

Los resultados del control de peso de los animales pertenecientes a cada lote fueron presentados y analizados para observar el comportamiento a lo largo del período de estudio (Tabla $2 \mathrm{y}$ Figura 1). Al día 30 de tratamiento, no se encontraron diferencias significativas de los 4 lotes ( $\mathrm{p}>$ 0,05) (Tabla 2), mientras que a los 60 días se observaron diferencias significativas entre el L1 y los tratamientos L2, L3 y L4 ( $\mathrm{p}=\mathrm{o}, 0013)$. Luego de 60 días se observó un descenso del peso vivo en los animales del grupo L3, mientras que el grupo testigo continuó en aumento, sin que se encontraran diferencias significativas entre los lotes a los 90 días ( $p>0,1667)$, aunque el L1 fue, de los grupos tratados, el que presentó un descenso más lento.

Tabla 2. Peso promedio en kilos de grupos de 5 bovinos de cada lote sometidos a 4 tratamientos con agua electroactivada en el agua de bebida.

\begin{tabular}{lllll}
\hline Lote $(\mathrm{n}=)$ & Día 0 & Día 30 & Día 60 & Día 90 \\
\hline $1(30)$ & $1337,3 \pm 34,5^{\mathrm{a}}$ & $1643,7 \pm 36,0^{\mathrm{a}}$ & $2088,3 \pm 250,8^{\mathrm{a}}$ & $2086,0 \pm 246,6^{\mathrm{a}}$ \\
$2(30)$ & $1354,0 \pm 41,9^{\mathrm{a}}$ & $1655,0 \pm 42,1^{\mathrm{a}}$ & $1814,0 \pm 51,5^{\mathrm{b}}$ & $1918,7 \pm 428,8^{\mathrm{a}}$ \\
$3(30)$ & $1349,0 \pm 44,1^{\mathrm{a}}$ & $1644,0 \pm 49,7^{\mathrm{a}}$ & $1821,0 \pm 47,6^{\mathrm{b}}$ & $1746,7 \pm 441,6^{\mathrm{a}}$ \\
$4(60)$ & $1374,2 \pm 46,7^{\mathrm{a}}$ & $1666,2 \pm 28,1^{\mathrm{a}}$ & $1875,2 \pm 53,4^{\mathrm{b}}$ & $2078,0 \pm 170,7^{\mathrm{a}}$ \\
\hline
\end{tabular}

aPromedio \pm desvío estándar. Letras distintas por columna indican diferencias estadísticamente significativas $(\mathrm{p}<0,05)$.

Tabla 3. Estadística descriptiva de los parámetros bioquímicos en sangre de bovinos en feedlot sometidos a tratamiento con agua electroactivada.

\begin{tabular}{lccccc}
\hline Lotes & 1 & 2 & 3 & 4 & $\mathrm{p}$ \\
\hline $\mathrm{pH}$ & $7,35 \pm 0,03$ & $7,32 \pm 0,09$ & $7,32 \pm 0,02$ & $7,38 \pm 0,03$ & 0,06 \\
$\mathrm{HCO} 3(\mathrm{mE} / \mathrm{L})$ & $26,18 \pm 1,13$ & $25,88 \pm 2,06$ & $23,9 \pm 3,97$ & $25,1 \pm 3,39$ & 0,64 \\
$\mathrm{Na}+(\mathrm{mmol} / \mathrm{L})$ & $144,20 \pm 4,32$ & $145,60 \pm 1,67$ & $145,00 \pm 2,55$ & $145,70 \pm 1,77$ & 0,74 \\
$\mathrm{~K}+(\mathrm{mmo} / \mathrm{L})$ & $4,46 \pm 1,26$ & $4,72 \pm 0,80$ & $5,58 \pm 1,15$ & $4,42 \pm 0,61$ & 0,15 \\
$\mathrm{Cl}-(\mathrm{mEq} / \mathrm{L})$ & $109,00 \pm 2,55$ & $109,40 \pm 1,67$ & $109,80 \pm 1,92$ & $109,40 \pm 1,35$ & 0,92 \\
$\mathrm{tHB}(\mathrm{g} / \mathrm{dL})$ & $13,52 \pm 0,53$ & $14,08 \pm 0,77$ & $12,30 \pm 3,01$ & $13,25 \pm 2,44$ & 0,61 \\
\hline
\end{tabular}

Los parámetros evaluados fueron: $\mathrm{pH}$, ( $\left.\mathrm{HCO}_{3}\right)$, sodio $(\mathrm{Na})$, potasio $(\mathrm{K})$, cloro $(\mathrm{Cl})$, hemoglobina total (tHB). Datos expresados en media +/- desvío estándar.

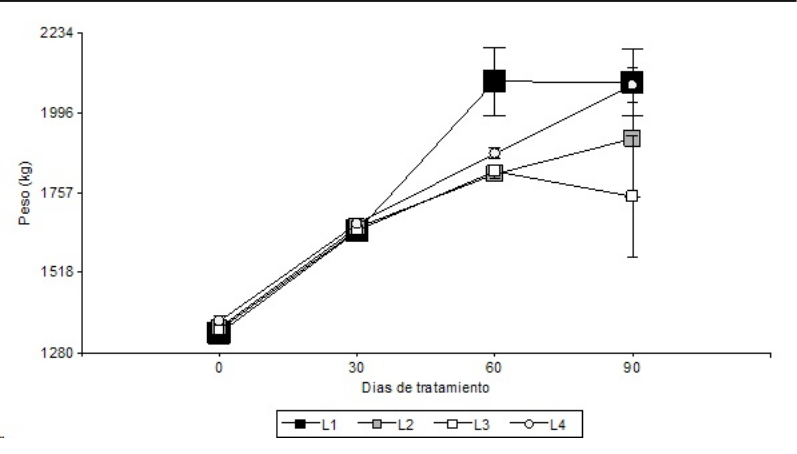

Figura 1. Gráfico de la progresión de los pesos por grupo de 5 animales de cada lote mes a mes (promedio \pm desvío estándar).

Las muestras de materia fecal resultaron positivas a E. coli O157 (stx2) en 3,3\% para el L2 a los 60 días y a Salmonella spp. en 3,3\% y 1,6\% para el L3 y el lote control a los 90 días, respectivamente.

Los resultados estadísticos descriptivos de los parámetros bioquímicos en sangre de los bovinos sometidos a tratamiento con $\mathrm{AE}$ se presentan en la Tabla 3 .

\section{Discusión}

Del análisis de los resultados de la administración de $\mathrm{AE}$ a bovinos de feedlot se desprenden varios puntos a considerar, entre ellos los referidos a la ganancia de peso. La tendencia a favor del L2 y L3 a los 60 días de tratamiento, podría deberse a un tipo de efecto promotor de crecimiento en los novillos por el consumo de $\mathrm{AE}$, aunque es necesario realizar estudios adicionales para corroborarlo, ya que el L1 que presentó mayor peso promedio fue el que recibió el tratamiento más prolongado. Los resultados de Bartolomé et al. (2011) e Ichinohe et al. (2004), indican que el $\mathrm{AE}$ podría tener un efecto sobre la actividad y eficacia de la microflora ruminal, lo que podría explicar el efecto promotor. De acuerdo con esto, el descenso de peso del L3, luego de los 60 días de tratamiento, coincide con la interrupción del consumo del AE 30 días antes. A partir del día 60 de ensayo, el L4 mantuvo un aumento de peso lineal sostenido alcanzando el peso final equivalente al L1, aunque resulta importante destacar que ese aumento fue más lento durante todo el ensayo. Por lo tanto, el tratamiento ininterrumpido con AE permitiría acortar el tiempo de terminación del animal y por ende reducir costos. De acuerdo con Tabernero de Paz et al. (2013), son escasos los trabajos que evalúen los efectos del $\mathrm{AE}$ sobre la productividad y rendimiento de los animales de abasto cuando se dosifica en el agua de bebida, siendo el presente trabajo de interés al respecto. Los resultados aquí presentados coinciden con estudios previos realizados en vacas lecheras, los cuales reportaron un aumento en la 
ganancia diaria de peso cuando se les suministró $\mathrm{AE}$ en el agua de bebida (D'Amico, 2003; Bartolomé et al., 2011).

De acuerdo con el Código Alimentario Argentino (CAA), cinco muestras de 65 y de 10 gramos de carne picada deben estar libres de $E$. coli O157 y Salmonella spp., respectivamente. Estas bacterias, que han sido reportadas en materia fecal de bovino en todo el mundo, pueden contaminar la carne durante la faena y procesado en frigorífico cuando el contenido intestinal contacta con la carcasa del animal portador. En el presente trabajo, se obtuvo 3,3\% de E. coli O157 para el L2 a los 60 días de tratamiento y el 3,3\% y 1,6\% de Salmonella spp. a los 90 días de tratamiento para los L3 y L4, respectivamente. La presencia de estas bacterias en la materia fecal de los novillos estudiados es inferior a la reportada por otros autores. Paiba et al. (2002) y Biruhtesfa et al. (2017) analizaron muestras de materia fecal de bovinos en frigoríficos de Gran Bretaña y Etiopía, obteniendo una prevalencia de E. coli O157 de 4,7 \% a 5,2 \%. En Estados Unidos y Venezuela se reportó una prevalencia de Salmonella en materia fecal de bovinos de 9,1 \% y 13,7 \% (Narváez-Bravo et al., 2013; Dargatz et al., 2016). Las variaciones en la prevalencia de estas bacterias patógenas pueden deberse a la categoría de animal, a la época del año durante la que se realiza la evaluación, al estrés del transporte asociado a un aumento de la eliminación, a factores dietarios o a la adición de AE en el agua de bebida (Ekong et al., 2015; Schuehle Pfeiffer et al., 2009; Dargatz et al., 2016). E. coli O157 y Salmonella spp. han sido asociadas a ETA en todo el mundo, siendo la carne uno de los alimentos implicados. Si bien los resultados obtenidos en el presente trabajo difieren de estudios previos, es necesaria la aplicación de buenas prácticas de higiene durante el proceso de faena para evitar la contaminación de la carne.

Un estudio previo en vacas lecheras concluye que el $\mathrm{AE}$ afecta al equilibrio ácido-base sanguíneo (Bartolomé et al., 2011). Sin embargo, en el presente trabajo no se evidenciaron alteraciones en los valores de referencia de los parámetros sanguíneos evaluados. Nuestros resultados coinciden con estudios realizados para evaluar los efectos del $\mathrm{AE}$ de diferentes $\mathrm{pH}$, cuando se dosifica en el agua de bebida a concentraciones variables en animales de laboratorio, perros y pollos (Inagaki et al., 2011; Morita et al., 2011; Yanagihara et al., 2005; Tabernero de Paz et al., 2013; Abol-Enein et al., 2009; Surdu et al., 2008).

\section{Conclusiones}

Se concluye que la adición de $\mathrm{AE}$ en el agua de bebida de bovinos de feedlot no produce alteraciones en los valores de referencia de los parámetros sanguíneos evaluados. De la misma manera, tampoco se evidenciaron modificaciones en la portación fecal de E. coli O157 y Salmonella spp. Se propone continuar esta investigación para comparar un tratamiento de 60 días al final del engorde para establecer si es la etapa más adecuada para su empleo como promotor de crecimiento, al mismo tiempo que podría compararse con un tratamiento equivalente a L1 a efectos de considerar cuál de ellos produce mayor beneficio en la disminución del tiempo necesario para terminar los animales. Por todo lo expuesto consideramos que, si bien es necesario profundizar las investigaciones en el uso del AE como promotora de crecimiento, los resultados obtenidos en el presente trabajo son alentadores y no deberían desestimarse.

\section{Conflicto de intereses}

Todos los autores declaran que no existen conflictos de intereses, incluyendo las relaciones financieras, personales o de otro tipo con otras personas u organizaciones que pudieran influir de manera inapropiada en el trabajo.

\section{Agradecimientos}

Los autores agradecen la colaboración de la Estancia La Verde de Blasfer S.A., partido de General Belgrano, Provincia de Buenos Aires, por su inestimable aporte para la realización del presente trabajo.

\section{Bibliografía}

Abol-Enein H, Gheith OA, Barakat N, Nour E, Sharaf AE. 2009. Ionized alkaline water: new strategy for management of metabolic acidosis in experimental animals. Therapeutic Apheresis and Dialysis. 13(3):220-4.

doi: 10.1111/j.1744-9987.2009.00659.x

Abu Aboud OA, Adaska JM, Williams DR, Rossitto PV, Champagne JD, Lehenbauer TW, Atwill R, Li X, Aly SS. 2016. Epidemiology of Salmonella sp. in California cull dairy cattle: prevalence of fecal shedding and diagnostic accuracy of pooled enriched broth culture of fecal samples. PeerJ. 30(4):e2386.

doi: $10.7717 /$ peerj.2386

Agga GE, Arthur TM, Schmidt JW, Wang R, Brichta-Harhay DM. 2016. Diagnostic accuracy of rectoanal mucosal swab of feedlot cattle for detection and enumeration of Salmonella enterica. Journal of Food Protection. 79(4):531-7. doi: 10.4315/0362-028X.JFP-15-409

Bartholomew ML, Heffernan RT, Wright JG, Klos RF, Monson T, Khan S, Trees E, Sabol A, Willems RA, Flynn R, Deasy MP, Jones B, Davis JP. 2004. Multistate outbreak of Salmonella enterica 
serotype enteritidis infection associated with pet guinea pigs. Vector-Borne and Zoonotic Diseases. 14(6):414-21.

doi: 10.1089/vbz.2013.1506

Bartolomé D, Posado R, Rodríguez L, Bueno F, Olmedo S, García JJ, Martín-Diana AB. 2011. Efecto higienizante del agua electrolizada sobre el agua de bebida y la calidad higiénico sanitaria de la leche. AIDA, XIV Jornadas sobre Producción Animal. Zaragoza. España. Tomo I:16-18.

Biruhtesfa A, Degmawi P, Mesele A, Genene T, Dereje H, Surafel K, Kebede A. 2017. Occurrence of Escherichia coli $\mathrm{O}_{157}: \mathrm{H}_{7}$ in cattle feces and contamination of carcass and various contact surfaces in abattoir and butcher shops of Hawassa, Ethiopia. BioMed Central Microbiology. 17:24.

doi: 10.1186/s12866-017-0938-1

Botta C, Ferrocino I, Cavallero MC, Riva S, Giordano M, Cocolin L. 2018. Potentially active spoilage bacteria community during the storage of vacuum packaged beefsteaks treated with aqueous ozone and electrolyzed water. International Journal of Food Microbiology. 266:337-45.

doi: 10.1016/j.ijfoodmicro.2017.10.012

Bouchrif B, Le Hello S, Pardos M, Karraouan B, Perrier-Gros-Claude JD, Ennaji MM, Timinouni M, Weill FX. 2009. Ceftazidime-resistant Salmonella enterica, Morocco. Emerging Infectious Diseases. 15(10):1693-5.

doi: 10.3201/eid1510.090247

Caprioli A, Morabito S, Brugere H, Oswald E. 2005. Enterohaemorrhagic Escherichia coli: emerging issues on virulence and modes of transmission. Veterinary Research. 36(3): 289-311. doi: 10.1051/vetres:2005002

Código alimentario argentino (Ley $\mathrm{N}^{\circ} 18284 / 69$ ), artículo 255. Capítulo VI. Alimentos cárneos y afines. CAA. Disponible en: http:// www.anmat.gov.ar/alimentos/codigoa/

Capitulo_VI_2017.pdf. Fecha de acceso: 20-12-17

Dargatz DA, Kopral CA, Erdman MM, FedorkaCray PJ. 2016. Prevalence and antimicrobial resistance of Salmonella isolated from cattle feces in United States feedlots in 2011. Foodborne Pathogen and Disease. 13(9):483-9.

doi: $10.1089 /$ fpd.2016.2128

Dean-Nystrom EA, Bosworth BT, Cray WC Jr, Moon HW. 1997. Pathogenicity of Escherichia coli $\mathrm{O} 157: \mathrm{H}_{7}$ in the intestines of neonatal calves. Infection and Immunity. 65(5):1842-8.

D'Amico, T. 2003. The drinking water facts. Health_BioNatural_The_Drinking_Water_FACT S.pdf. Disponible en: http://members.iinet.net.au/ $\sim$ sambrod/ . Fecha de acceso: 19/12/17.
Ekong PS, Sanderson MW, Cernicchiaro N. 2015. Prevalence and concentration of Escherichia coli O157 in different seasons and cattle types processed in North America: A systematic review and meta-analysis of published research. Preventive Veterinary Medicine. 121(1-2):74-85. doi: 10.1016/j.prevetmed.2015.06.019

Envirolife. Instalación del primer equipo de Aguas Electroactivadas en Catamarca. Disponible en: http://envirolife.com.ar/aguas-electroactivadasequipo/. Fecha de acceso: 19-12-17

European Food Safety Authority (EFSA). Scientific report of EFSA. 2009. Technical specifications for the monitoring and reporting of verotoxigenic Escherichia coli (VTEC) on animals and food (VTEC surveys on animals and food). doi: 10.2903/j.efsa.2009.1366

Ferens WA, Hovde CJ. 2011. Escherichia coli O157:H7: animal reservoir and sources of human infection. Foodborne Pathogen and Disease. 8(4): 465-87.

doi: 10.1089/fpd.2010.0673

Ferguson JD, Remsberg D, Wu Z. 2008. Influence of electrolyzed alkaline water on milk production in dairy cows. Journal of Animal Science. 91:609.

Fey PD, Safranek TJ, Rupp ME, Dunne EF, Ribot E, Iwen PC, Bradford PA, Angulo FJ, Hinrichs SH. 2000. Ceftriaxone-resistant Salmonella infection acquired by a child from cattle. The New England Journal of Medicine. 342(17):1242-9.

doi: 10.1056/NEJM200004273421703

FSIS. 2017. Directive 7120.1. Rev. 43. Safe and suitable ingredients used in the production of meat, poultry, and egg products.

Herikstad H, Motarjemi Y, Tauxe RV. 2002. Salmonella surveillance: a global survey of public health serotyping. Epidemiology \& Infection. 129(1):1-8.

doi: 10.1017/So950268802006842

Holcroft JJMS. 2003. Effect of anolyte on broiler performance. Tesis de Maestría de Filosofía. Universidad de Stellenbosch. South Africa.

Hughes P, Heritage J. Antibiotic growthpromoters in food animals. Disponible en: http:// www.fao.org/docrep/ARTICLE/AGRIPPA/

555_EN.HTM. Fecha de acceso: 19-12-17.

Ichinohe T, Gotou M, Fujihara T. 2004. Influences of basic electrolyzed water on water absorption, ruminal environment, microbial yield and nitrogen balance in sheep. Animal Science Journal. 75: 67-76.

Inagaki H, Shibata Y, Obata T, Kawagoe M, Ikeda 
K, Sato M, Toida K. 2011. Effects of slightly acidic electrolysed drinking water on mice. Laboratory Animals, 45(4):283-5.

doi: 10.1258/la.2011.010122

Karmali MA, Gannon V, Sargeant JM. 2010. Verocytotoxin-producing Escherichia coli (VTEC). Veterinary Microbiology. 140(3-4):360-70. doi: 10.1016/j.vetmic.2009.04.011

Leotta GA, Chinen I, Epszteyn S, Miliwebsky E, Melamed IC, Motter M. 2005. Validation of a multiplex PCR for detection of Shiga toxinproducing Escherichia coli. Revista Argentina de Microbiología. 37(1):1-10.

Morita C, Nishida T, Ito K. 2011. Biological toxicity of acid electrolyzed functional water: Effect of oral administration on mouse digestive tract and changes in body weight. Archives Oral Biology. 56(4):359-66.

doi: 10.1016/j.archoralbio.2010.10.016

Narváez-Bravo C, Miller MF, Jackson T, Jackson S, Rodas-Gonzalez A, Pond K, Echeverry A, Brashears MM. 2013. Salmonella and Escherichia coli $\mathrm{O}_{157} \mathrm{H} 7$ prevalence in cattle and on carcasses in a vertically integrated feedlot and harvest plant in Mexico. Journal of Food Protection. 76(5):78695.

doi: 10.4315/0362-028X.JFP-12-079

O'Brien SB, Duffy G, Carney E, Sheridan JJ, McDowell DA, Blair IS. 2005. Prevalence and numbers of Escherichia coli O157 on bovine hides at a beef slaughter plant. Journal of Food Protection. 68(4):660-5.

Paiba GA, Gibbens JC, Pascoe SJ, Wilesmith JW, Kidd SA, Byrne C, Ryan JB, Smith RP, McLaren M, Futter RJ, Kay AC, Jones YE, Chappell SA, Willshaw GA, Cheasty T. 2002. Faecal carriage of verocytotoxin-producing Escherichia coli O157 in cattle and sheep at slaughter in Great Britain. Veterinary Record. 150(19): 593-8.

Perry JJ, Yousef AE. 2011. Decontamination of raw foods using ozone-based sanitization techniques. Annual Review of Food Science and Technology. 2: 281-98.

doi: 10.1146/annurev-food-022510-133637

Ramoneda M, Foncuberta M, Simón M, Sabate S, Herrera S, Landa B, Musté N, Martí R, Trabado V, Carbonell O, Vila M, Espelt M, Ramírez B, Durán
J. 2013. Prevalence of verotoxigenic Escherichia coli O157 (VTEC O157) and compliance with microbiological safety standards in bovine carcasses from an industrial beef slaughter plant. Letter in Applied Microbiology. 56(6):408-13.

doi: 10.1111/lam.12062

Roy R, Higgins R, Fortin M, Tardif S. 2001. Salmonella give infection in 2 dairy herds. Canadian Veterinay Journal. 42(6):468-70.

Smith AB, Renter DG, Cernicchiaro N, Shi X, Nagaraja TG. 2016. Prevalence and quinolone susceptibilities of Salmonella isolated from the feces of preharvest cattle within feedlots that used a fluoroquinolone to treat bovine respiratory disease. Foodborne Pathogen and Disease. 13(6): 303-8.

doi: 10.1089/fpd.2015.2081

Surdu I, Vatuiu I, Jurcoane S, Ciocîrlan A. 2008. Modern methods ensuring sanitary veterinary protection for animal farms, based on using of electrolyzed water. 7th International Symposium of Animal Nutrition and Biology. Balotesti. Rumania. 25-26 September. Disponible en: http:// www.envirolytecanada.com/wp-content/uploads/ 2015/03/EnvrioNize-Envirolyte-Poultry-CaseStudy-Abstract-Modern-methods-sanitaryveterinary-protection-using-ELECTROLYZEDWATER-Romania-s.pdf. Fecha de acceso: 19-12-17

Tabernero de Paz MJ, Bodas R, Bartolomé D, Posado R, García JJ, Olmedo S. 2013. Agua electrolizada como higienizante en producción animal: efectos en sanidad y productividad. Revista Archivos de Zootecnia. 62(R):13-23.

Venkitanarayanan KS, Ezeike GO, Hung YC, Doyle MP. 1999. Efficacy of electrolyzed oxidizing water for inactivating Escherichia coli O157:H7, Salmonella enteritidis, and Listeria monocytogenes. Applied and Environmental Microbiology. 65(9):4276-9.

Wray C, Wary A. 2000. Salmonella in domestic animals. Wallingford, CABI Publishing.

Yanagihara T, Arai K, Miyamae K, Sato B, Shudo T, Yamada M, Aoyama M. 2005. Electrolyzed hydrogen-saturated water for drinking use elicits an antioxidative effect: a feeding test with rats. Bioscience Biotechnology Biochemistry. 69(10): 1985-7.

doi: 10.1271/bbb.69.1985 